\title{
Consideraciones para fortalecer la vinculación universitaria en territorios rurales. Reflexiones con base en el estudio de sistemas de producción
}

\section{Considerations in order to strengthen university social work in rural territories. Reflections based on the study of production systems}

CHRISTIAN VICENTE TAMAYO ORTIZ(*) (D),DORIS PILAR CUICHAN CATAGÑA (b) @D, VINICIO GERMÁN AYALA RODRÍGUEZ (c) (D), MANUEL MARÍA PUMISACHO GUALOTO(a)(D, GUSTAVO FERNANDO SEVILLANO VASQUEZ(a)(D)

\author{
(a) Universidad Central del Ecuador. Facultad de Ciencias Agrícolas. \\ Jerónimo Leiton y Av. La Gasca s/n. Ciudadela Universitaria 17052.1, Quito - Ecuador \\ (b) Asesora Agrícola Independiente, Quito - Ecuador \\ (c) Municipio del Distrito Metropolitano de Quito, Av. 24 de Mayo y Manuel E. Silva E1 -25, Puéllaro - Quito - Ecuador. \\ *cvtamayo@uce.edu.ec
}

\section{RESUMEN}

En la parroquia Puéllaro se identificó la dinámica agraria y el modelo actual de vinculación con la sociedad con el objetivo de generar algunas consideraciones que se puedan aplicar para fortalecer el proceso impulsado en este territorio. Para el efecto, se realizaron recorridos de campo, entrevistas y estudios de caso en fincas, considerando tipologías de productores. Las actividades son realizadas mediante la articulación de dos actores: universidad y gobierno local en el marco de un

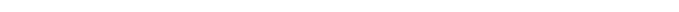

proyecto de servicio comunitario. El principal sector intervenido se sitúa en áreas bajas y cálidas con producción frutícola; sin embargo, el análisis agrario evidenció la existencia de otras zonas agroecológicas con características productivas distintas, problemáticas más complejas y condiciones de vulnerabilidad de la población. Las pautas para fortalecer el trabajo promovido están relacionadas con: (i) Considerar la diversidad agroecológica y tipología de productores; (ii) Estructurar equipos 
interdisciplinarios; (iii) Promover mayor participación de líderes comunitarios y productores; y (iv) Explorar otras líneas de acción, especialmente a través de la investigación y educación continua. La realidad campesina identificada, en relación al limitado acceso a la tierra, carencia de riego y bajos ingresos agrícolas, puso en evidencia la necesidad de articulación con otros actores con competencia en el sector rural. Los elementos generados constituyen una guía para fortalecer el servicio a la colectividad en el sector rural y orientar la formulación de propuestas más amplias.

Palabras clave: Servicio comunitario, zonificación agroecológica, diversidad productiva, ingresos agrícolas, articulación de actores.

\section{ABSTRACT}

In the Puéllaro parish, the agricultural dynamics and the current model of social work with the society were identified with the aim of generating some reflections that can be applied to strengthen the process promoted in this territory. For this purpose, different field trips, interviews, and case studies were carried out on farms based on several types of producers. The activities are carried out through the articulation of two elements: university and local government within the framework of a community service project. The main sector intervened is located in the low and warm areas with fruit production; however, the agricultural analysis showed the existence of other agroecological zones with different productive characteristics, having more complex problems and conditions of vulnerability of the population. The guidelines to strengthen the promoted work are related to the following aspects: (i) To consider the agroecological diversity and types of producers; (ii) To organize interdisciplinary teams; (iii) To promote more participation of community leaders and producers; and, (iv) To explore other lines of action, especially through research and continuing education. The identified reality of this indean community in relation to the limited access to land, lack of irrigation, and low agricultural income, evidenced the need for coordination with other actors with responsibilities in the rural sector. The elements generated constitute a guide to strengthen the service to the community in the rural sector and in this way to guide the formulation of broader proposals.

Keywords: Community service, Agroecological zoning, productive diversity, agricultural income, articulation of actors.

\section{DESCRIPCIÓN DEL PROBLEMA SOCIAL}

En Puéllaro se desarrolla un trabajo de vinculación entre la Facultad de Ciencias Agrícolas de la Universidad Central del Ecuador y la Delegación Norcentral del Gobierno Municipal del Distrito Metropolitano de Quito. A pesar de los esfuerzos realizados, se puede identificar un débil accionar a escala territorial por la temporalidad de las acciones, falta de articulación con otros actores, escasa participación campesina; e insuficiente comprensión de las dinámicas agrarias. Estas particularidades influyen a concentrar el apoyo en un sector determinado y no poder expandir las acciones a zonas con mayor vulnerabilidad. Por tal motivo, es indispensable fortalecer el proceso implementado. 


\section{SÍNTESIS Y APLICACIONES PRÁCTICAS}

- El análisis de los sistemas de producción a nivel parroquial puso en evidencia la diversidad de zonas agroecológicas existentes, tipos de productores, orientación productiva y condiciones socioeconómicas de las familias. Esta información constituye un marco de referencia para definir escenarios de vinculación y formulación de propuestas tomando en cuenta la realidad y dinámica de cada espacio territorial. Además de servir como indicadores de línea base para futuras evaluaciones.

- Se establecen algunas pautas que se pueden aplicar a fin de fortalecer el proceso de vinculación en la zona; además de servir de referencia para trabajos en otros territorios rurales.

- Se identificó las condiciones socioeconómicas que enfrenta la producción campesina en relación con el limitado acceso a la tierra, carencia de riego y bajos ingresos agrícolas; poniendo en evidencia que para su mejoramiento es importante la articulación de otros actores con competencia en el sector rural.

\section{INTRODUCCIÓN}

En la última década, las Instituciones de Educación Superior (IES) se han sumado al esfuerzo de solventar demandas de la sociedad mediante una mayor proyección en su accionar (Barreno et al., 2018). Las principales actividades para solventar necesidades y desafíos del entorno están relacionadas con la investigación, proyectos de servicio comunitario, educación continua, prácticas profesionales, entre otras acciones relevantes (Consejo de Educación Superior [CES], 2019).

Sin lugar a duda, el sector académico constituye un actor de trascendental importancia en el ámbito del desarrollo socioeconómico y bienestar de la población (Bajo, 2013), tanto en el sector urbano como en el rural; por lo tanto, es preciso reflejar las condiciones en las que ejecuta actividades de relacionamiento o más conocidas como vinculación con la sociedad. Al respecto, diversos trabajos reflejan la importancia del vínculo de las universidades, tanto para beneficio de la sociedad (Aguilar-Rodríguez, 2015; Araya-Chaves, B., \& Barboza-Flores, S., 2015; Giobergia \& Cereceto, 2016; Hinojosa, 2016; Viña et al., 2019) como para la formación profesional y humanísticas de sus estudiantes (Monge-González et al., 2016; Mendoza et al., 2017; Zambrano et al., 2018); sin embargo, también han empezado a reflejar sus limitaciones. Las debilidades estarían relacionadas con la visión de corto plazo de los proyectos y falta de empoderamiento con la problemática (Viña et al., 2019); ausencia de metodologías apropiadas para el trabajo con actores sociales (Araya-Chaves, B., \& Barboza-Flores, S., 2015); escasa articulación entre docencia, investigación y vinculación (Barreno et al., 2018); inexistencia de estudios técnicos adecuados que reflejen las necesidades de los sectores rurales, e insuficiente participación de estudiantes y docentes (Mendoza et al., 2017). A esta realidad se suman la ausencia de promoción, reconocimiento y prioridad de esta actividad al interior de las IES; así como también, el escaso presupuesto asignado (Simbaña-Cabrera \& Correa, 2017). Adicionalmente, varios estudios plantean el impulso de modelos alternativos de gestión que mejoren 
la calidad de la intervención (Vizcaíno, 2013; Polaino \& Romillo, 2017; Simbaña-Cabrera \& Correa, 2017).

A pesar de que los trabajos realizados plantean la necesidad de mejoras en los procesos desarrollados, no evidencian el complejo escenario en los que se insertan las entidades académicas relacionadas con territorios rurales. Por tal motivo, es preciso evidenciar la forma como se la implementa en áreas agropecuarias; y en base al contraste con las dinámicas agrarias que se suscita en su interior, reflejar los principales desafíos y elementos que deben ser considerados para el impulso de nuevas estrategias de intervención.

En este marco se inscribe el estudio realizado en un territorio de la serranía ecuatoriana, específicamente en la parroquia Puéllaro; espacio donde una entidad académica pública, juntamente con un Gobierno Autónomo Descentralizado Municipal (GAD-M) realizan procesos de servicio a la comunidad. El objetivo del estudio fue generar nuevos elementos que permitan fortalecer el proceso de vinculación efectuado en esta zona, poniendo en evidencia las dinámicas agrarias en base a la caracterización de los sistemas de producción existentes. De manera específica, se identificó las principales características del modelo de vinculación implementado; y a su vez, visibilizó la existencia de nuevos escenarios de trabajo en base a la diversidad de zonas agroecológicas, sistemas productivos y realidad campesina. Estos aspectos constituyen insumos para estructurar un nuevo esquema de intervención en vías de coadyuvar esfuerzos con otros actores públicos y privados para mejorar las condiciones de la población rural.

\section{MATERIALES Y MÉTODOS}

La investigación se desarrolló en la parroquia Puéllaro. Esta localidad está ubicada en la zona nororiental del cantón Quito, provincia de Pichincha, Ecuador; cubre un rango altitudinal entre los 1650 a 3400 msnm y presenta una diversidad de climas, topografía, suelos, vegetación natural y cultivada.

Fue realizada considerando tres etapas: (i) Identificación del modelo actual de vinculación en la zona; (ii) Análisis de la microrregión con principios sistémicos; y, (iii) Definición de nuevos elementos para fortalecer el proceso de servicio con la sociedad.

\section{(i) Identificación del modelo actual de vinculación}

A partir de recorridos preliminares por toda la parroquia, diálogo con actores y revisión de informes de actividades, se identificaron los principales elementos del modelo actual de vinculación impulsado en el territorio. Los aspectos reflejados están relacionados con las áreas geográficas de intervención, actores relacionados con el proceso de trabajo, líneas impulsadas, frecuencia de intervención, entre otros aspectos relevantes.

\section{(ii) Análisis de la microrregión con principios sistémicos}

Fue desarrollado según el marco metodológico para el análisis de un territorio rural con principios sistémicos planteados por Apollín \& Eberhart (1999) y Cochet et al., (2002). Este enfoque permitió analizar la parroquia desde una perspectiva integral para evidenciar la diversidad de realidades que se presentan en su interior. A su vez, consideró las siguientes etapas: 


\section{Identificación de áreas con dinámicas homogéneas}

La identificación delimitó áreas territoriales homogéneas con relación a características biofísicas y agroecológicas (uso y tipo de suelo, clima, topografía, principales cultivos, entre otros). Fue imprescindible el análisis de cartografía digital y fotografías aéreas; además de recorridos por la parroquia haciendo uso de la técnica de lectura del paisaje y elaboración de transectos para entender como está configurado el espacio agrario (Cochet et al., 2002). Finalmente, se estableció una tipología de zonas agroecológicas y su delimitación con el software ARGIS 10.3.

\section{Caracterización de sistemas productivos en base a tipolo- gías de productores}

Para el análisis de sistemas productivos se realizaron 36 estudios de caso haciendo uso de una ficha estructurada. La selección de productores fue a través de una muestra razonada no probabilística en base a casos representativos de cada una de las categorías planteadas en las distintas zonas identificadas. Finalmente, la información fue concentrada tomando en cuenta los grupos establecidos y se estructuró una categorización definitiva considerando aspectos productivos y socioeconómicos.

(iii) Planteamiento de consideraciones para el fortalecimiento del proceso de vinculación

Se contrastó la realidad identificada con el modelo de intervención, y se estructuró las principales consideraciones para el impulso y fortalecimiento del proceso de vinculación.

\section{RESULTADOS}

Principales elementos del modelo de vinculación implementado

El proceso de vinculación impulsado en la parroquia Puéllaro está configurando por el accionar de dos actores: (i) La academia, con participación de estudiantes de los últimos semestres de la carrera de ingeniería agronómica encargados de implementar las actividades de campo planificadas, conjuntamente con un docente tutor que coordina las prácticas, emite informes y evalúa el trabajo estudiantil; y (ii) el Gobierno Municipal, a través de un técnico local que coordina las acciones en territorio y que avala el cumplimiento de las actividades realizadas. En lo que respecta al rol de la comunidad, esta se limita a la participación en charlas o visitas a sus predios sin un rol preponderante al momento de planificar las actividades y evaluar los trabajos realizados.

Con relación al tipo de acciones impulsadas, en el marco de un convenio realizado entre las dos instancias, se ha privilegiado el componente de vinculación denominado "servicio comunitario", a través de la formulación e implementación de un proyecto con la misma denominación.

La principal área de intervención constituye una zona frutícola ubicada en la parte baja de la parroquia y en sitios cercanos al poblado central de Puéllaro; mientras que los actores comunitarios que se insertan en el trabajo impulsado constituyen agricultores individuales con una lógica de producción frutícola, con acceso al riego y poseedores de terrenos ubicados en áreas planas a ondu-

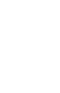


Consideraciones para fortalecer la vinculación universitaria en territorios rurales. Reflexiones con base en el estudio de sistemas de producción - Christian Vicente Tamayo, Doris Pilar Cuichan, Vinicio Germán Ayala, Manuel María Pumisacho, Gustavo Fernando Sevillano • VÍNCULOS-ESPE (2021) VOL.6, No.3: 13-29

ladas. Las acciones realizadas giran en torno al establecimiento de un huerto frutal demostrativo en el cerro La Luz en base a durazno (Prunus persica), aguacate (Persea americana), chirimoya (Annona cherimola), entre otras especies; poda de frutales, manejo de plagas, entre otras actividades ligadas con la producción.

La frecuencia de intervención es un día a la semana (aproximadamente cinco horas de trabajo efectivo en campo) durante un semestre académico; tiempo en el cual, el grupo de estudiantes asignados al territorio cumplen 160 horas que acreditan su trabajo como requisito para su proceso de graduación. Al respecto, los estudiantes emiten al docente coordinador un informe de actividades realizadas y expresan el grado de satisfacción con las prácticas realizadas, resultados obtenidos y acompañamiento recibido por parte del docente tutor en base a una escala de valoración que va desde mala a excelente.

\section{Zonas agroecológicas identificadas}

Puéllaro presenta al menos cinco zonas agroecológicas que reflejan las distintas realidades que afronta la población campesina en el sector rural (Tabla 1). En la zona se identificó espacios con ecosistema natural (Bosque y vegetación seca herbácea) y áreas agrícolas con dinámicas productivas diversas. Las partes medias a altas de la parroquia se caracterizan por la carencia de riego, relieve irregular y enfrentar procesos erosivos muy fuertes por las prácticas de labranza implementadas; mientras que las áreas más bajas poseen una topografía ondulada a suave y cuentan con acceso al recurso hídrico.
Tabla 1

Principales características de las zonas agroecológicas identificadas en la parroquia Puéllaro

\begin{tabular}{|c|c|}
\hline Zona & Características \\
\hline $\begin{array}{c}\text { Bosque } \\
\text { protector (A) }\end{array}$ & $\begin{array}{l}\text { Ecosistema ubicado en la parte alta de la } \\
\text { parroquia (Sobre los } 3100 \text { m.s.n.m.). Su } \\
\text { gestión es comunal. }\end{array}$ \\
\hline $\begin{array}{l}\text { Territorio } \\
\text { maicero con } \\
\text { pastizales (B) }\end{array}$ & $\begin{array}{l}\text { Parte media-alta de la parroquia (2 } 700 \text { a } 3 \\
100 \text { m.s.n.m.). Superficie alrededor de } 16.7 \\
\text { km2 (37,4 \% del territorio agrícola). Zona } \\
\text { carente de riego, con relieve pronunciado y } \\
\text { susceptible a la erosión. }\end{array}$ \\
\hline $\begin{array}{l}\text { Área frutícola } \\
\text { y de cultivos } \\
\text { de ciclo (C) }\end{array}$ & $\begin{array}{l}\text { Zona media-baja (2 } 100 \text { a } 2700 \text { m.s.n.m.). } \\
\text { Con } 9.6 \mathrm{~km} 2 \text { ( } 21,5 \% \text { del territorio). Posee } \\
\text { un relieve moderado a fuerte, clima } \\
\text { templado y existencia de predios con y sin } \\
\text { acceso al riego. }\end{array}$ \\
\hline $\begin{array}{l}\text { Área frutícola } \\
\text { con huertas } \\
\text { tradicionales (D) }\end{array}$ & $\begin{array}{l}\text { Zona baja (2 } 000 \text { a } 2100 \text { m.s.n.m.), Cubre } \\
2.4 \mathrm{~km} 2 \text { ( } 5,4 \% \text { del territorio). Presenta clima } \\
\text { cálido, relieve con inclinaciones leves en el } \\
\text { fondo del valle y acceso al riego. } \\
\text { Actualmente, es el espacio y escenario } \\
\text { principal del proceso de vinculación. }\end{array}$ \\
\hline $\begin{array}{l}\text { Área frutícola } \\
\text { con huertas } \\
\text { tradicionales } \\
\text { y especies } \\
\text { subtropicales (E) }\end{array}$ & $\begin{array}{l}\text { Áreas más bajas de la parroquia ( } 1700 \text { a } 2 \\
000 \text { m.s.n.m.). Superficie de } 3.1 \text { km2 (cubre } \\
6.9 \% \text { del territorio). Clima cálido con } \\
\text { acceso al riego. }\end{array}$ \\
\hline
\end{tabular}

Nota. Elaborado por los autores con base en recorridos de campo, análisis de cartografía y delimitación en software ARGIS. 
La producción agrícola también es variada. La Zona B, con clima templado y mayor vulnerabilidad a la erosión, es valorizada principalmente para el desarrollo del cultivo de maíz (Zea mays) y crianza de bovinos. Las áreas más bajas, cálidas y con riego (Zona C, D y E) son aprovechadas para el desarrollo de producción hortofrutícola con algunas particularidades. En la zona $C$ se desarrollan cultivos de ciclo corto y huertas frutales, los predios pueden o no tener acceso al riego. El área frutícola con huertas tradicionales (Zona D) presenta mayoritariamente aguacate (Persea americana), chirimoya (Annona cherimola), cítricos, entre otros. Finalmente, la zona $\mathrm{E}$ (área frutícola con huertas tradicionales y especies subtropicales), al tener un clima más cálido presenta cultivos como plátano (Musa paradisiaca), papaya (Carica papaya), yuca (Manihot esculenta), uva (Vitis spp.), mango (Mangifera indica) y varias especies hortícolas. Adicionalmente, es posible evidenciar áreas relacionadas con agricultura empresarial y hacendataria (producción florícola, láctea y crianza de aves de postura) categorizadas como "no estudiadas" por no ser objeto de este estudio y no tener a la presente un nexo con el servicio a la sociedad.

La organización del paisaje y zonas agroecológicas identificadas se aprecian en la Figura 1.

Figura 1

Zonas agroecológicas identificadas en la parroquia Puéllaro
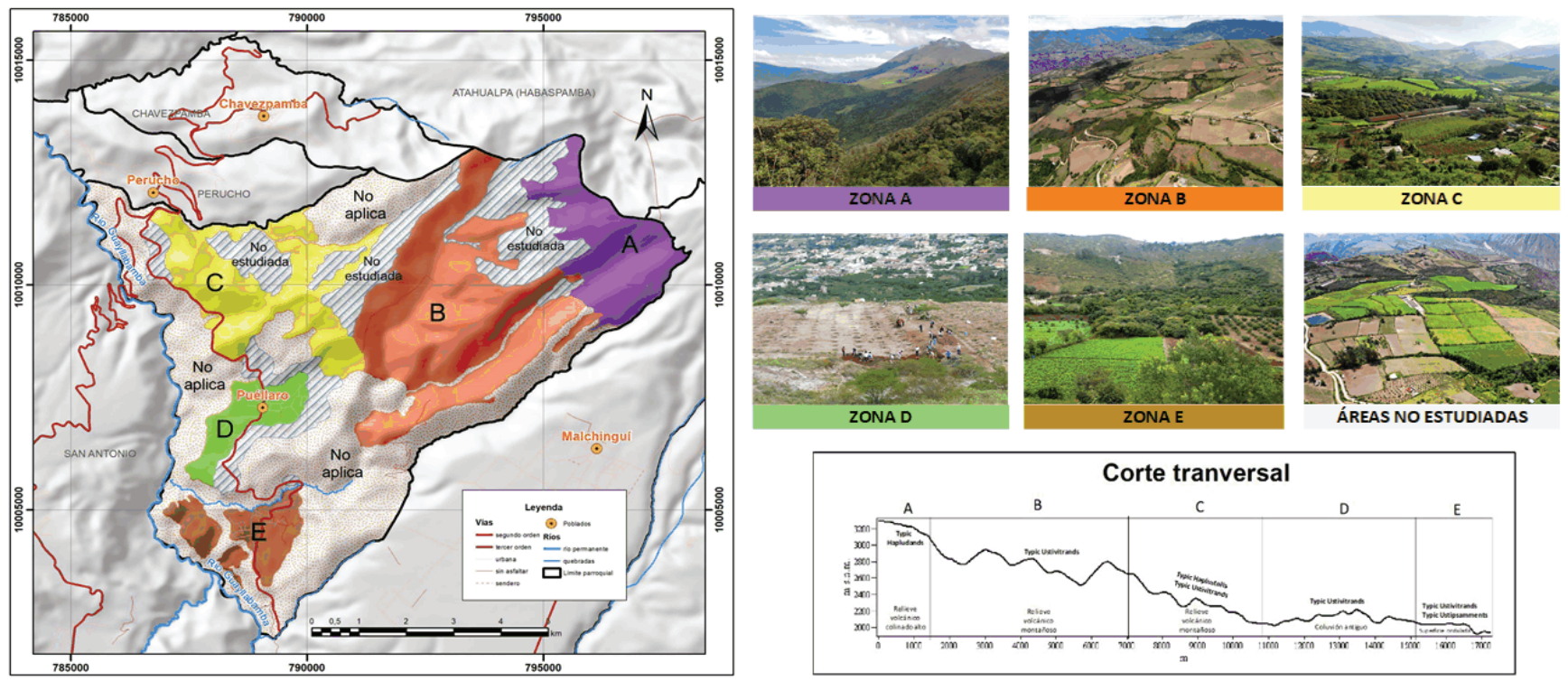

Nota. Elaborado con base en recorridos de campo, cartografía base OpenStreetMap (OSM) 2021, límites cantonales Comité Nacional de Límites Internos (CONALI) 1:50.000, 2019 y delimitación de zonas agroecológicas en software ARGIS. 
Consideraciones para fortalecer la vinculación universitaria en territorios rurales. Reflexiones con base en el estudio de sistemas de producción - Christian Vicente Tamayo, Doris Pilar Cuichan, Vinicio Germán Ayala, Manuel María Pumisacho, Gustavo Fernando Sevillano • VÍNCULOS-ESPE (2021) VOL.6, No.3: 13-29

\section{Tipología de productores y su orientación productiva}

La diversidad de áreas territoriales identificadas es acompañada de la existencia de diferentes tipos de sistemas productivos. De los estudios de casos realizados, se estructuró seis tipos de sistemas productivos con diferente acceso a la tierra, ubicación agroecológica, orientación productiva y acceso al riego (Tabla 2). Todos los campesinos tienen la característica de poseer varios rubros en la finca combinando cultivos (ciclo corto, frutales y/u hortalizas) con crianzas (mayores o menores).

Los productores de las zonas media a alta de la parroquia tienen una mayor orientación a la siembra de maíz; sin embargo, según la disponibilidad de terreno, crían animales para producción de leche (Tipo A) o para el engorde (Tipo B). A nivel de fruticultores, es posible diferenciar a campesinos que poseen en sus predios únicamente especies frutales con variación en el acceso a la tierra (Tipos C y D) y productores que adicionalmente implementan cultivos hortícolas como el tomate (Lycopersicum sculentum) y cultivos de ciclo corto como el fréjol (Phaseolus vulgaris), (Tipo E y F). Adicionalmente, un aspecto común entre la mayor parte de las categorías es la existencia de especies menores en sus fincas como cuyes, gallinas y cerdos de engorde, con variación en la cantidad de animales.

\section{Disponibilidad y destino de la fuerza de trabajo familiar}

Todas las categorías de agricultores destinan una parte importante de su mano de obra en actividades fuera de su finca, con mayor énfasis en campesinos que no poseen acceso al riego (maiceros)
Tabla 2

Sistemas productivos identificados en la parroquia Puéllaro

\begin{tabular}{|c|c|c|}
\hline $\begin{array}{l}\text { Tipos de } \\
\text { productores* }\end{array}$ & $\begin{array}{l}\text { Acceso } \\
\text { la tierra }\end{array}$ & $\begin{array}{l}\text { Orientación } \\
\text { productiva }\end{array}$ \\
\hline $\begin{array}{l}\text { Maiceros con crianzas } \\
\text { mayores para leche } \\
\text { (Tipo A). }\end{array}$ & $\begin{array}{l}2 \text { a } 8 \text { ha en } \\
\text { áreas frágiles } \\
\text { sin riego }\end{array}$ & \multirow{2}{*}{$\begin{array}{l}\text { Principalmente cultivo de } \\
\text { maíz y cría de bovinos. } \\
\text { Adicionalmente, especies } \\
\text { menores como cerdos, cuyes } \\
\text { y gallinas de traspatio en } \\
\text { menor cantidad. }\end{array}$} \\
\hline $\begin{array}{l}\text { Maiceros con crianzas } \\
\text { mayores para engorde } \\
\text { (Tipo B). }\end{array}$ & $\begin{array}{l}<2 \text { ha en } \\
\text { áreas frágiles } \\
\text { sin riego }\end{array}$ & \\
\hline $\begin{array}{c}\text { Medianos frutícultores } \\
\text { con crianzas menores } \\
\text { (Tipo C). }\end{array}$ & $\begin{array}{l}2 \text { a } 5 \text { ha en } \\
\text { áreas onduladas } \\
\text { con o sin riego }\end{array}$ & \multirow{2}{*}{$\begin{array}{l}\text { Huertas frutales (aguacate, } \\
\text { chirimoya, cítricos, entre } \\
\text { otros). Adicionalmente } \\
\text { crianzas menores como } \\
\text { cuyes, gallinas de traspatio o } \\
\text { cerdos en mayor cantidad. }\end{array}$} \\
\hline $\begin{array}{l}\text { Pequeños frutícultores } \\
\text { con crianzas menores } \\
\text { (Tipo D). }\end{array}$ & $\begin{array}{c}<2 \text { ha en } \\
\text { áreas de valle } \\
\text { con riego }\end{array}$ & \\
\hline $\begin{array}{l}\text { Pequeños fruticultores } \\
\text { horticultores } \\
\text { (Tipo E). }\end{array}$ & $\begin{array}{c}<2 \text { ha en } \\
\text { áreas de valle } \\
\text { con riego }\end{array}$ & \multirow{2}{*}{$\begin{array}{l}\text { Huertas frutales (aguacate, } \\
\text { chirimoya, entre otros) y } \\
\text { hortalizas como tomate y } \\
\text { fréjol. Las crianzas son } \\
\text { escasas y se limitan a pocos } \\
\text { animales como cerdos. }\end{array}$} \\
\hline $\begin{array}{l}\text { Medianos fruticultores } \\
\text { horticultores } \\
\text { (Tipo F). }\end{array}$ & $\begin{array}{l}>5 \text { ha en } \\
\text { áreas de valle } \\
\text { con riego }\end{array}$ & \\
\hline
\end{tabular}

Nota. Elaborado con base en el análisis de los estudios de caso realizados. Se excluyen sistemas basados en el cultivo de flores y crianzas de gallinas ponedores por no ser objeto de este estudio.

y fruticultores con limitado acceso a la tierra (Tipos D y E), (Tabla 3). En contraste, productores con mayor acceso a terreno, orientan su mano de obra en más del $80 \%$ para la actividad agrícola; e 
incluso, el sistema productivo puede demandar la contratación de mano de obra permanente. En lo que respecta a la edad de las cabezas de hogar, bordea los 50 años y es catalogada como una población adulta.

\section{Tabla 3}

Disponibilidad y destino de la mano de obra familiar por tipo de productor en la parroquia Puéllaro

\begin{tabular}{cccccccccc}
$\begin{array}{c}\text { Categorías } \\
\text { de }\end{array}$ & $\begin{array}{c}\text { Superficie } \\
\text { productores }\end{array}$ & $\begin{array}{c}\text { Edad } \\
\text { (años) }\end{array}$ & \multicolumn{2}{c}{$\begin{array}{c}\text { UTF } \\
\text { Total }\end{array}$} & \multicolumn{2}{c}{$\begin{array}{c}\text { UTF } \\
\text { finca }\end{array}$} & \multicolumn{2}{c}{$\begin{array}{c}\text { UTF } \\
\text { fuera-finca }\end{array}$} \\
\cline { 8 - 10 } A & 4,5 & 54 & 3,2 & 100 & 2,0 & 64,2 & 1,1 & 35,8 \\
B & 1,2 & 47 & 3,3 & 100 & 2,0 & 60,1 & 1,3 & 39,9 \\
C & 3,5 & 52 & 1,7 & 100 & 1,6 & 94,1 & 0,1 & 5,9 \\
D & 0,6 & 46 & 2,2 & 100 & 1,3 & 59,7 & 0,9 & 40,3 \\
E & 1,4 & 53 & 2,1 & 100 & 1,2 & 57,1 & 0,9 & 42,9 \\
F & 8,7 & 50 & 3,5 & 100 & 2,9 & 82,9 & 0,6 & 17,1 \\
\hline
\end{tabular}

Nota. UTF = Unidades de Trabajo Familiar. Elaborado con base en el análisis de los estudios de caso realizados.

\section{Ingresos campesinos}

El ingreso generado por la actividad agrícola varía según tipo de productor, identificándose remuneraciones superiores o inferiores al salario de un obrero agrícola local (15USD/día). La dificultad económica por la que atraviesan los campesinos se hace visible en aquellas categorías situadas en territorios sin riego y con fuertes procesos erosivos (Categorías A y B) y con predios reducidos en zonas frutícolas (Categoría D). En contraste, los agricultores fruticultores con predios mayores a las tres hectáreas (Categoría C y F) obtienen los mejores ingresos, sobre los 20USD/día. Caso particular constituyen los fruticultores-horticultores con predios por debajo de las dos hectáreas (Tipo D), que logran obtener un valor que bordea los 20 USD/día, favorecidos por el acceso al riego y la actividad intensiva hortícola, (Tabla 4).

\section{Tabla 4}

Ingresos campesinos según categoría de productores en la parroquia Puéllaro

\begin{tabular}{|c|c|c|c|c|c|c|}
\hline \multirow{2}{*}{ Variables } & \multicolumn{6}{|c|}{ Categoría de productores } \\
\hline & $\mathbf{A}$ & B & c & D & $\mathbf{E}$ & $\mathbf{F}$ \\
\hline Superficie (ha) & 4,5 & 1,2 & 3,5 & 0,6 & 1,4 & 8,7 \\
\hline $\begin{array}{c}\text { Ingreso Total (IT) } \\
\text { USD/año }\end{array}$ & 8761 & 10008 & 9643 & 9181 & 8689 & 25103 \\
\hline$\%$ & 100 & 100 & 100 & 100 & 100 & 100 \\
\hline $\begin{array}{l}\text { Ingreso agrícola neto } \\
\text { (IAN) USD/año }\end{array}$ & 3771 & 2076 & 8935 & 1619 & 6289 & 23503 \\
\hline$\%$ & 43 & 21 & 93 & 18 & 72 & 94 \\
\hline $\begin{array}{l}\text { Otros ingresos } \\
\text { (OI) USD/año }\end{array}$ & 4991 & 7932 & 708 & 7562 & 2400 & 1600 \\
\hline$\%$ & 57 & 79 & 7 & 82 & 28 & 6 \\
\hline IAN/día (USD/día) & 8 & 5 & 25 & 6 & 24 & 37 \\
\hline IT/día (USD/día) & 13 & 14 & 26 & 19 & 19 & 33 \\
\hline
\end{tabular}

Nota. Elaborado con base en el análisis de los estudios de caso realizados. 
Consideraciones para fortalecer la vinculación universitaria en territorios rurales. Reflexiones con base en el estudio de sistemas de producción - Christian Vicente Tamayo, Doris Pilar Cuichan, Vinicio Germán Ayala, Manuel María Pumisacho, Gustavo Fernando Sevillano • VÍNCULOS-ESPE (2021) VOL.6, No.3: 13-29

El nivel de remuneración generado motiva a las familias a la búsqueda y obtención de otras fuentes de ingresos en actividades asalariadas fuera de su hogar, como en el sector florícola y de servicios. Los rubros económicos obtenidos por otras vías (OI), representan entre el 50 y el $80 \%$ de los ingresos familiares para el caso de productores de ciclo corto (Categorías A y B) y campesinos fruticultores con predios reducidos (Categoría D).
Consideraciones para impulsar un nuevo esquema de intervención

En virtud del contraste entre la dinámica territorial y el modelo actual de intervención se identificaron los principales elementos que no están siendo tomados en cuenta al momento de realizar el proceso de vinculación con la sociedad en la localidad; y que, con el fin de fortalecer su proceso, se conciben como consideraciones (Figura 2).

Figura 2

Consideraciones al momento de intervenir en el territorio en el marco de procesos de vinculación con la sociedad

TERRITORIO

A. CONSIDERAR LA DIVERSIDAD EXISTENTE Y GENERACIÓN DE PROPUESTAS DIFERENCIADAS

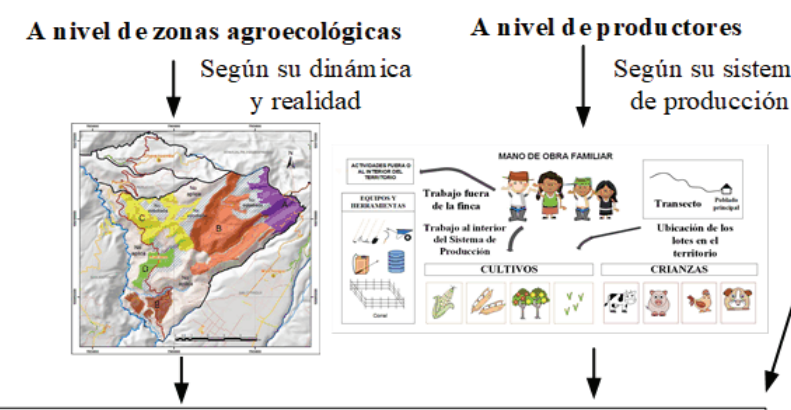

\begin{tabular}{|c|c|}
\hline A nivel de territorio & A nivel de UPA \\
\hline $\begin{array}{lr}\text { Conservación de Recursos } \\
\text { Naturales (Suelos, Agua, } \\
\text { otros); Fortalecimiento de la } \\
\text { organización comunitaria; } \\
\text { entre otras reciones } \\
\text { relevantes. }\end{array}$ & $\begin{array}{l}\text { Manejo de suelos, agua, } \\
\text { cultivos, crianzas, } \\
\text { transformación de productos, } \\
\text { actividades no agrícolas; entre } \\
\text { otras acciones relevantes. }\end{array}$ \\
\hline
\end{tabular}

ACTORES

\section{B. ARTICULACIÓN CON ACTORES}

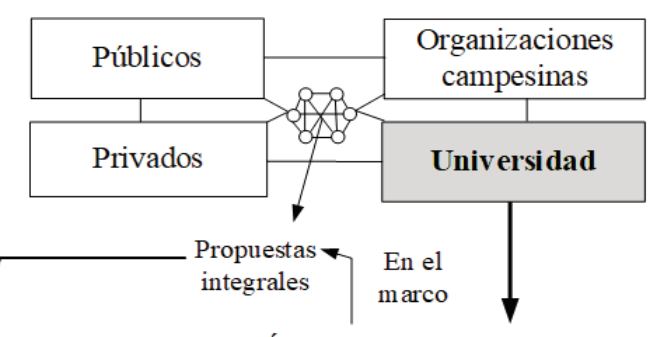

C. IMPLEMENTACIÓN DE PROPUESTAS CONSIDERANDO

EJES DE VINCULACIÓN

\begin{tabular}{|c|c|c|}
\hline Investigación & Docentes & Estudiantes \\
\hline $\begin{array}{c}\text { Servicio } \\
\text { comunitario }\end{array}$ & \multirow{3}{*}{$\begin{array}{l}\text { Formulación de } \\
\text { propuestas, } \\
\text { acompañamiento } \\
\text { a estudiantes, } \\
\text { asesoría en } \\
\text { territorio, otros. }\end{array}$} & \multirow{3}{*}{$\begin{array}{l}\text { Ejecución de } \\
\text { actividades } \\
\text { con objetivo } \\
\text { de doble vía }\end{array}$} \\
\hline $\begin{array}{l}\text { Prácticas pre } \\
\text { profesionales }\end{array}$ & & \\
\hline $\begin{array}{c}\text { Educación } \\
\text { continua }\end{array}$ & & \\
\hline $\begin{array}{r}\text { Conocimiento } \\
\text { conocimien }\end{array}$ & $\begin{array}{l}\text { idad, aplicación } \\
\text { ndizaje vivencia }\end{array}$ & \\
\hline
\end{tabular}

Nota. Elaborado con base en el contraste entre la dinámica agraria identificada y el esquema actual de intervención. 
De manera global se destaca la necesidad de: (i) Tomar en cuenta la diversidad existente y su dinámica tanto a nivel de zonas agroecológicas como de sistemas productivos para impulsar propuestas diferenciadas acorde a la realidad de cada área y tipo de productor; (ii) Fortalecer el vínculo de trabajo con otros actores del territorio, tanto públicos como privados, donde se destaque una participación más activa de la comunidad; e, (iii) Implementar propuestas tomando en cuenta ejes adicionales de vinculación como investigación, prácticas pre-profesionales y educación continua en el marco de propuestas integrales a nivel más global.

Finalmente se puede destacar que los estudiantes que se insertan en el sector, además de cumplir con actividades planificadas, fortalezcan su comprensión sobre la dinámica del territorio rural y de las condiciones que favorecen o limitan el desarrollo de la agricultura.

\section{DISCUSIÓN}

Existencia de una diversidad productiva y la necesidad de una intervención y formación integral

El territorio analizado presenta una diversidad de zonas agroecológicas, sistemas productivos y problemáticas de carácter integral; sin embargo, se ha privilegiado el accionar únicamente en un sector determinado (área frutícola cercana al poblado central). Esta particularidad se originó al momento de definir el alcance del convenio suscrito y etapa de concepción del proyecto basado en la identificación de problemas, definición de objetivos y establecimiento de actividades sin contar con un estudio preliminar que muestre la dinámica agraria de toda la parroquia; además no se refleja una participación efectiva de los productores y articulación con otros actores, tanto al interior de la propia entidad académica como de otras esferas gubernamentales con injerencia a nivel local.

A fin de fortalecer la intervención realizada, es necesario ampliar el actual radio de acción, garantizar la participación comunitaria y definir escenarios de vinculación en base a las características agroecológicas, socioeconómicas y de los diferentes tipos de productores existentes, y no únicamente en las características de la carrera universitaria que se inserta en la localidad y el perfil del o los docentes que liderarán el proceso de servicio a la sociedad. Además, demanda de los actores que se insertan en la localidad, conocimientos teóricos y prácticos en diversos ámbitos profesionales (Ecología, organización comunitaria, manejo de suelos, técnicas de riego y conservación de humedad, producción de especies de ciclo corto, perenne, crianza de animales mayores, menores, turismo, entre otros temas relevantes). Por tal motivo, la formación integral en el campo de las ciencias agropecuarias resulta de vital importancia para fortalecer las actividades que realizan las entidades académicas en territorios rurales; aspecto que concuerda con lo planteado por Malagón (2016) que lo resalta como fundamental para impulsar mejoras en la sociedad.

Adicionalmente, la estructuración de equipos interdisciplinarios para la concepción e implementación de propuestas integrales constituiría un desafío para los procesos de vinculación impulsados por las Instituciones de Educación Superior (Araya- 
Chaves \& Barboza-Flores, 2015; Baca-Tavira \& Herrera-Tapia, 2016; Brito-Gaona et al., 2018); aspecto que fortalecería las capacidades locales del grupo de trabajo que intervendría en este proceso.

\section{Trabajo con población adulta y que orienta su mano de} obra a la pluriactividad

Los resultados evidencian un sector campesino caracterizado por una edad alrededor de los 50 años, y que destina buena parte de su fuerza de trabajo a actividades extra prediales; aspecto que coincide con otros estudios sobre sistemas de producción campesinos (Tamayo et al., 2015). Esta realidad demanda a docentes y estudiantes, el manejo de metodologías de trabajo para interactuar con diversos grupos etarios. Además, plantea la necesidad de interrelacionarse con los miembros de familia que tengan una gestión directa con la finca, sobre todo, al momento de definir los períodos de tiempo más adecuados para fomentar acciones de vinculación con la sociedad.

Mejoramiento de las condiciones de vida de la población rural: ¿̇ás allá del alcance de las universidades?

Uno de los principales desafíos en el sector rural en general y en esta microrregión en particular, constituye el mejoramiento de las condiciones socioeconómicas de las familias con menor acceso a recursos productivos (tierra y agua). Esta realidad aflora la necesidad de plantear las siguientes interrogantes: ¿̇Hasta qué punto el mejoramiento de las condiciones económicas de los campesinos debe ser considerado como una de las finalidades de la vinculación con la sociedad?; y ¿̇El mejoramiento de la calidad de vida de las familias es un aspecto que puede sobrepasar el accionar de las universidades? Sin tratar de dar una respuesta específica a cada incógnita, es preciso resaltar que si bien las universidades deben: "[...] responder a las expectativas y necesidades de la sociedad [...]" (Brito-Gaona et al., 2018, p. 47) y constituirse en actores fundamentales para impulsar el desarrollo y bienestar de la sociedad (Figueroa-Sepúlveda, 2003; Bajo, 2013), con énfasis en los estratos más vulnerables de la sociedad (Polaino \& Romillo, 2017; Malagón, 2016); con los resultados evidenciados es posible afirmar que propiciar un mejoramiento en las condiciones que atraviesa la población campesina demanda también la articulación e intervención de otros actores con competencias en la formulación e implementación de políticas y propuestas integrales en el sector agrario a nivel nacional, provincial y local.

En efecto, como lo señala la Comunidad de Estados Latinoamericanos y Caribeños [CELAC] y Organización de las Naciones Unidas para la Alimentación y la Agricultura [FAO] (2017), la problemática del sector rural demanda superar la lógica de intervenciones aisladas que no constituirían en una herramienta eficaz para enfrentar la pobreza del campo. Además, de la necesidad de intervenciones integrales donde la articulación de actores (Hall et al., 2005; Monge-González et al., 2016; Barreno et al., 2018); y sobre todo la calidad de sus interacciones (Ortiz et al., 2013), constituyan sus principales estrategias de acción.

En este contexto, las entidades académicas deben constituirse en un actor adicional encaminado a contribuir al desarrollo integral de un territorio rural, en el marco de sus competencias y fortalezas. 
Por las razones señaladas, el servicio a la sociedad impulsado por las universidades con vínculo en el sector rural, en la medida de lo posible debe inscribirse en procesos más amplios de planificación e intervención. De lo contrario, se corre el riesgo de pensar que el sector académico debe asumir la responsabilidad social que le corresponde al Estado (Barreno et al., 2018).

\section{Intervención considerando varios ejes de acción}

El proceso vinculación con la sociedad enmarca varias líneas de acción como servicio comunitario, educación continua, prácticas pre-profesionales, investigación, entre otras actividades relevantes (CES, 2019). Sin embargo, en el territorio analizado se considera una sola estrategia "el servicio comunitario", realidad que concuerda con lo identificado por Rueda et al. (2020) al evidenciar el predominio de la acción social en las actividades de vínculo con la sociedad de las IES ecuatorianas. En este marco, los estudiantes y el tutor o tutores ingresan y salen del territorio de manera itinerante hasta que se cumpla un determinado número de horas, requisito formal para la graduación estudiantil, originando rupturas en la intervención e inacción en territorio durante el período intersemestral.

A fin de fortalecer el proceso de servicio a la sociedad y dar respuestas más específicas frente a la compleja problemática que enfrenta la sociedad rural, resultaría de utilidad el impulso de los distintos ejes de intervención en el territorio con una estrategia de continuidad; sobre todo, concebir a la investigación y educación continua como herramientas sustanciales para afianzar el vínculo con el sector campesino. Al respecto, varios autores destacan la importancia de la articulación entre investigación y vinculación para mejorar las actividades de relacionamiento con la sociedad (Vizcaíno, 2013; Bajo, 2013; Monge-González et al., 2016; Barreno et al., 2018; Brito-Gaona et al., 2018); además, de la necesidad de enfocarla a sectores sociales que implementan sistemas productivos complejos y con base territorial (Coraggio, 2002). Sin embargo, no se destaca a los procesos de capacitación como actividad indispensable para el nexo de la academia con los sectores campesinos, muy probablemente debido a que buena parte de las IES del país no consideran esta acción como de vinculación con la sociedad (Rueda et al., 2020).

\section{La exploración del territorio y el aprendizaje vivencial}

Finalmente, es necesario destacar que, en un vínculo de trabajo conjunto entre la academia y la sociedad rural todos sus actores deben fortalecer sus capacidades producto de las acciones realizadas. Si bien varios autores resaltan la importancia de este proceso para beneficio de los actores universitarios (profesionales y estudiantes) en relación a su formación e incremento de conocimientos (Vizcaíno, 2013; Araya-Chaves \& Barboza-Flores, 2015; Monge-González et al., 2016; Brito-Gaona et al., 2018), es vital reflexionar acerca del proceso de aprendizaje vivencial de los estudiantes antes, durante y después de implementar un trabajo conjunto con los agricultores. Es indispensable que conozcan la diversidad del territorio, problemática, historia de formación, sistemas productivos existentes, calendarios agrícolas, entre otros aspectos relevantes; además, durante el trabajo en las fincas, es necesario que se identifique la composición familiar, acceso a recursos productivos, itinerario técnicos de cultivos y crianzas, lógica de implementación de prácticas agropecuarias, 
entre otros; y, al finalizar su accionar, haber fortalecido sus destrezas técnicas en producción, habilidades de trabajo con agricultores y comprender los factores que favorecen o limitan el desarrollo de la agricultura en un entorno determinado. Por estas razones resultaría fundamental evaluar el nivel de aprendizaje vivencial adquirido por los estudiantes en el marco anteriormente señalado.

\section{CONCLUSIONES}

En la parroquia Puéllaro se ha implementado un modelo de vinculación con la sociedad sustentado en el sector académico y Gobierno Municipal; la estrategia de intervención impulsada ha sido el servicio comunitario, mediante el accionar de estudiantes de los últimos niveles de la carrera de Ingeniería Agronómica, un docente tutor y el apoyo de un coordinador técnico local municipal. La principal área de intervención constituye el área frutícola con limitada participación de productores y líderes campesinos; sin embargo, se identificó varias zonas agroecológicas, diversidad de sistemas productivos y condiciones socioeconómicas contrastantes que reflejan la necesidad de fortalecer el esquema actualmente desarrollado.
Con base en las particularidades identificadas es pertinente impulsar nuevas estrategias de intervención considerando la diversidad territorial, metodologías de trabajo con población adulta, mayor participación comunitaria, articulación con varios actores, varias líneas de vinculación y el fortalecimiento del aprendizaje vivencial de los estudiantes.

\section{AGRADECIMIENTOS}

Un profundo agradecimiento a la Universidad Central del Ecuador por financiar la realización de la presente investigación ejecutada en el marco del proyecto "Identificación de los Agroecosistemas de las provincias de Pichincha y Cotopaxi; y caracterización de los sistemas de producción en el área de influencia directa de la Facultad de Ciencias Agrícolas", proyecto №37; y a la Delegación Norcentral de la Administración Norte Eugenio Espejo del Municipio del Distrito Metropolitano de Quito por el acompañamiento en todas las etapas de este estudio.

\section{REFERENCIAS}

Aguilar-Rodríguez, I. (2015). La vinculación universitaria con la sociedad desde una perspectiva ecuatoriana. Revista Economía y Negocios, 6(2), 62-68. https://doi. org/10.29019/eyn.v6i2.305

Apollin, F., \& Eberhart, C. (1999). Análisis y diagnóstico de los sistemas de producción en el medio rural. Guía metodológica. Sistema de Capacitación para el Manejo de los Recursos Naturales Renovables [CAMAREN].

Araya-Chaves, B., \& Barboza-Flores, S. (2015). Mejora de la capacidad competitiva de asociaciones de productores de granos. En C. Garrido-Noguera., \& D. García-Pérez (coords.) Vinculación de las universidades con los sectores productivos. Casos en Iberoamérica, vol. 1 (p.p. 21 30). Ciudad de México, México: Unión de Universidades 
de América Latina y el Caribe, A. C. [UDUAL]., \& Red Universidad-Empresa América Latina y El Caribe-Unión Europea [REDUE-ALCUE].

Baca-Tavira, N., \& Herrera-Tapia, F. (2016). Proyectos sociales. Notas sobre su diseño y gestión en territorios rurales. Convergencia, 23(72), 69-87.

Bajo, A. (2013). Universidad Autónoma de Sinaloa. Su contribución al desarrollo regional. En M. Martínez., F. Piñero., \& S. Figueroa (coords.) El papel de la universidad en el desarrollo (p.p. 67-76). Benemérita Universidad Autónoma de Puebla \& Universidad Nacional del Centro de la Provincia de Buenos Aires.

Barreno-Salinas, M., Barreno-Salinas, Z., \& Olmedo-Valencia, A. C. (2018). La educación superior y su vinculación con la sociedad: referentes esenciales para un cambio. Universidad y Sociedad, 10(1), 40-45.

Brito-Gaona, L., Quezada-Abad, C., \& Gordillo-Quizhpe, I. (2018). La vinculación con la sociedad y la universidad pública en el Ecuador. En L. Brito-Gaona., C. QuezadaAbad., \& L. Arzola de la Rosa (coords.) La universidad y la vinculación con la sociedad. Una condición impostergable (p.p. 28-53). Universidad Técnica de Machala [UTMACH].

Cochet, H.; Brochet, M.; Ouattara, Z.; \& Boussou, V. (2002). Démarche d'étude des systèmes de production de la région de Korhogo-Koulokakaha-Gbonzoro en Côte d'Ivoire. Les éditions du GRET.

Comunidad de Estados Latinoamericanos y Caribeños [CELAC] \& Organización de las Naciones Unidas para la Alimentación y la Agricultura [FAO]. (2017). Sistemas de innovación para el Desarrollo Rural Sostenible. CELAC. http://www.fao. org/3/i7769s/i7769s.pdf

Consejo de Educación Superior [CES]. (2019). Reglamento de Régimen Académico. C.E.S. Gaceta Oficial. http://gaceta. ces.gob.ec.

Coraggio, J.L. (2002, 23 y 24 de julio). Universidad y desarrollo local [Ponencia] Seminario Internacional La educación superior y las nuevas tendencias, Quito, Ecuador.

Figueroa-Sepúlveda, V. (2013). El rol de las universidades en el Desarrollo. La perspectiva de los organismos internacionales. En M. Martínez., F. Piñero., \& S. Figueroa (coords.) El papel de la universidad en el desarrollo (p.p. 11-22). Benemérita Universidad Autónoma de Puebla \& Universidad Nacional del Centro de la Provincia de Buenos Aires.

Giobergia, C., \& Cereceto, M. F. (2016). Desarrollo de nuevos productos a través de la extracción de principios activos de plantas: en caso de la alcaparra en Santiago del Estero. En C. Garrido-Noguera., \& D. García-Pérez (coords.) Vinculación de las universidades con los sectores productivos. Casos en Iberoamérica, vol. 1 (p.p. 57-66). Unión de Universidades de América Latina y el Caribe, A. C. [UDUAL]., \& Red Universidad-Empresa América Latina y El Caribe-Unión Europea [REDUE-ALCUE].

Hall, A., Mytelka, L., \& Oyeyinka, B. (2005). Innovation system: Implications for agricultural policy and practice. ILAC. Brief (2), 1-4. 
Hinojosa, C. (2016). Vinculación con la sociedad una función estratégica de la universidad. Revista vínculos, 1 (1), 3-5.

Malagón, L. (2006). La vinculación Universidad-Sociedad desde una perspectiva social. Educación y Educadores, 9(2), 79-93.

Mendoza-Macías, C.A., Jácome-Vélez, T. G., \& Vélez-Loor, M.E. (2017). La Participación de la Comunidad Universitaria y su Vinculación con la Sociedad. Revista Científica Hallazgos 21, 2(3), 212- 221.

Monge-González, M., Segreda-Rodríguez, A., Campos-Meléndez, L., \& Chaves-Abarca. R. (2016). Vinculación para el desarrollo del cultivo, industrialización y comercialización de guayabita de Perú, en un asentamiento campesino de la Estrella. En C. Garrido-Noguera., \& D. García-Pérez (coords.) Vinculación de las universidades con los sectores productivos. Casos en Iberoamérica, vol. 1 (p.p. 77-85). Unión de Universidades de América Latina y el Caribe, A. C. [UDUAL]., \& Red Universidad-Empresa América Latina y El Caribe-Unión Europea [REDUE-ALCUE].

Ortiz, O., Orrego, R., Pradel, W., Gildemacher, P., Castillo, R., Otiniano, R., Gabriel, J., Vallejo, J., Torres, O., Woldegiorgis, G., Damene, B., Kakuhenzire, R., Kasahija, I., \& Kahiu, I. (2013). Insights into potato innovation systems in Bolivia, Ethipia, Peruand Uganda.Agricultural Systems, $114(1), 73: 83$. https://doi.org/10.1016/j.agsy.2012.08.007

Polaino, C. \& Romillo, A. (2017). Vinculación con la Sociedad en la Universidad de Otavalo, Ecuador. Formación Universitaria, 10(3):21-30. https://dx.doi.org/10.4067/ S0718-0062017000300004
Rueda, I., Acosta, B., \& Cueva, F. (2020). Las universidades y sus prácticas de vinculación con la sociedad. Educação \& Sociedade, (41),1-16. https://dx.doi.org/10.1590/ es. 218154

Simbaña-Cabrera, H. \& Correa, L. (2017). La vinculación con la sociedad en el Ecuador: Reflexiones sobre su itinerario curricular. Revista vínculos, 2(1), 5-12.

Tamayo, C., Ortiz, R., \& Cepeda, D. (2017). Sistemas de producción campesinos y gestión social del riego: el caso de la acequia Mocha-Huachi. Siembra, 4(1), 21-30. https:// doi.org/10.29166/siembra.v4i1.299

Viña, N., Sacoto, V., \& Landívar, J. (2019). Contribución de la Universidad ecuatoriana en beneficio de sectores vulnerables-Vinculación con la Sociedad. Revista Espacios 40(23), 1-11.

Vizcaíno, J. (2013). Diseño de un modelo de gestión para la vinculación de las instituciones de educación superior con la comunidad. [Tesis de maestría, Universidad Central del Ecuador], Repositorio Institucional. DSpace. http://www. dspace.uce.edu.ec/

Zambrano, T., Molina, P., Murillo, A., González, O., \& Batista, A. (2018). Impacto de los proyectos de vinculación en la formación profesional y humanística de los estudiantes de la Universidad San Gregorio de Portoviejo. Revista San Gregorio, (24), 50-59. 


\section{BIOGRAFÍA DE LOS AUTORES}

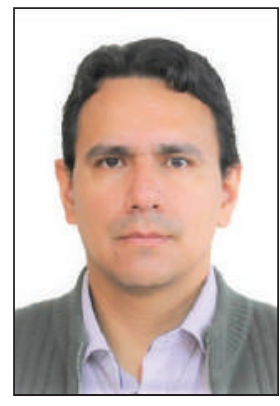

\section{CHRISTIAN TAMAYO}

Ingeniero Agrónomo. Docente de la Facultad de Ciencias Agrícolas de la Universidad Central del Ecuador. Experiencia profesional en docencia universitaria, capacitación campesina, proyectos de desarrollo rural e investigación en dinámicas agrarias, sistemas productivos y gestión integral del riego. Ex becario de la unidad ABC del Distrito Metropolitano de Quito para estudios de posgrado en Economía Agrícola y Desarrollo Sustentable; AGCl-Chile en Manejo Integrado de Cuencas Hidrográficas, Desarrollo Sustentable de Territorios Rurales y Medio Ambiente; y, del programa Cochran en Agricultura Resiliente en Carolina del Norte-EEUU. Actualmente cursa el Doctorado en Agricultura Sustentable en la Universidad Agraria La Molina en Perú.

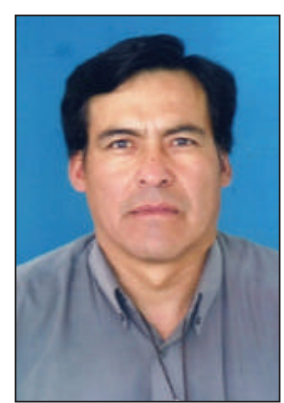

\section{MANUEL PUMISACHO}

Ingeniero Agrónomo graduado en la Facultad de Ciencias Agrícolas de la Universidad Central del Ecuador. Realizó estudios de posgrado en el Colegio de Pos Graduados de México donde obtuvo el título de Maestro en Ciencias Especialista en Desarrollo Rural. Durante 24 años vinculado al Instituto Nacional Autónomo de Investigaciones Agropecuarias (INIAP) en el área de Validación y Transferencia de Tecnología. Coautor y autor de 11 publicaciones relacionadas con el cultivo papa y herramientas de aprendizaje. Desde marzo del 2007 hasta la actualidad docente de la Facultad de Ciencias Agrícolas de la Universidad Central del Ecuador en Cultivos y Extensión Agropecuaria.

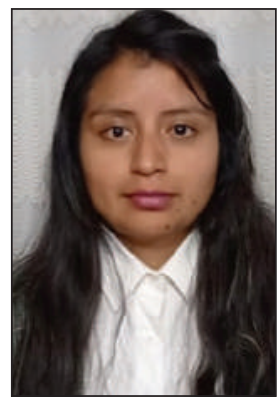

\section{DORIS CUICHAN}

Ingeniera Agrónoma gradudada en la Facultad de Ciencias Agrícolas de la Universidad Central del Ecuador con el mérito de mejor egresada en el año 2019. Su trabajo de titulación estuvo relacionado con el análisis de sistemas de producción en la parroquia Puéllaro. Desarrolla actividades relacionadas con la horticultura, fruticultura y asesoría en desarrollo de cultivos intensivos.

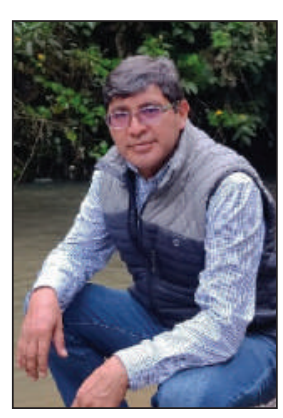

\section{VINICIO AYALA}

Ingeniero Agroforestal con maestría en Desarrollo Rural. Gestor cultural y emprendedor. Responsable de Gestión participativa y del desarrollo en la Delegación Municipal Norcentral del Distrito Metropolitano de Quito. Coordinador del proyecto Cerro La Luz en la parroquia Puéllaro. Generador de proyectos de producción y turismo sostenible.

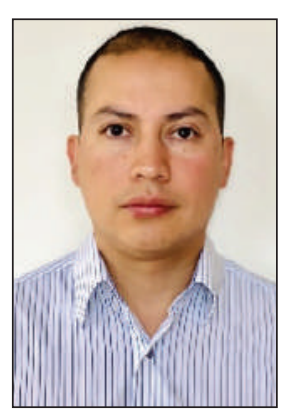

\section{GUSTAVO SEVILLANO}

Docente de la cátedra de Sistemas de Información Geográfica (SIG) en la Facultad de Ciencias Agrícolas. Investigador en el campo de Edafología Coautor del libro The Soils of Ecuador, capítulo Soil from the Coastal Plane publicado Springer International Publishing. Coautor del Atlas de suelos de América Latina y el Caribe para la Comisión Europea Oficina de Publicaciones de la Unión Europea. Consultor para generación de geoinformación

\author{
para el desarrollo
}

